\title{
DECREASED GROWTH WITH SEROTONIN REUPTAKE INHIBITORS
}

Growth and growth hormone secretion were evaluated in 4 children (3 boys), aged 11.6 to 13.7 years, treated with selective serotonin reuptake inhibitors (SSRIs) for obsessive compulsive disorder or Tourette syndrome, at Schneider Children's Medical Center of Israel, Petah Tikva, and Sackler School of Medicine, Tel Aviv University, Tel Aviv. SSRIs (fluvoxamine, fluoxetine) were administered for 6 months to 5 years (dosage, $20-100 \mathrm{mg} /$ day) and patients were examined for height, bone age, pubertal progression, and hypothalamic-pituitary function. Growth retardation occurred in all 4, and in 3 when a growth spurt was anticipated. Three had a decreased growth hormone response to clonidine stimulation, and 1 had decreased 24-hour secretion of growth hormone that became normal after SSRI treatment was discontinued. Two resumed normal growth when therapy was discontinued, and in 2 patients somatropin was added to the SSRI regimen. (Weintrob N, Cohen D, Klipper-Aurbach Y, Zadik Z, Dickerman Z. Decreased growth during therapy with selective serotonin reuptake inhibitors. Arch Pediatr Adolesc Med July 2002;156:696-701). (Reprints: Naomi Weintrob MD, Institute for Endocrinology and Diabetes, Scneider Children's Medical Center of Israel, 14 Kaplan St, Petah Tikva 49202, Israel).

COMMENT. A decrease in growth rate and delayed puberty may occur during treatment with SSRI for various psychiatric disorders in children. Suppression of growth hormone secretion is suspected. Further studies are indicated, but the use of SSRIs in children should be reevaluated and carefully monitored. Caution is especially important if SSRIs are used in children with ADHD, in combination with methylphenidate or amphetamines, drugs with proven growth retardation potential.

\section{RANDOMIZED, CONTROLLED STUDY OF ADDERALL XR IN ADHD}

The efficacy and safety of Adderall XR in the treatment of attention deficit/hyperactivity disorder and diurnal variation in responses were assessed by a multicenter, randomized, double-blind, parallel group, placebo-controlled trial at 47 sites, and reported from the Massachusetts General Hospital, Boston, MA. Patients received single-daily morning doses of placebo or Adderall 10, 20, or 30 $\mathrm{mg}$ for 3 weeks. In 509 children aged 6 to 12 years who completed the study, Intention-to-treat analysis of Conners Global Index Scales for Teachers and Parents revealed significant improvement in morning, afternoon, and late afternoon behavior for all active treatment groups versus placebo, significant dose-related improvements in behavior from baseline, and all doses were superior to placebo. Adverse events that occurred significantly more frequently with Adderall than placebo included anorexia, in $22 \%$ of participants, insomnia (17\%), abdominal pain (12\%), and emotional lability (9\%). Anorexia was dose-related. The majority (69\%) of adverse events were mild, $28 \%$ moderate, and $4 \%$ were severe. Nine Adderall-treated patients required withdrawal of medication because of anorexia, abdominal pain, or nausea. Laboratory values including ECG showed no significant changes. (Biederman J, Lopez FA, Boellner SW, Chandler MC. A randomized, double-blind, placebo-controlled, parallel-group study of SL1381 (Adderall XR) in children with attention-deficit/hyperactivity disorder. Pediatrics August 2002;110:258-266). (Reprints: Joseph Biederman MD, Pediatric Psychopharmacology Unit, Massachusetts General Hospital, 15 Parkman St-ACC 725, Boston, MA 02114).

COMMENT. It is reassuring to know that the effectiveness of Adderall XR noted in clinical practice is confirmed in controlled trial. 